\title{
Dynamic Mechanism of Photochemical Induction of Turing Superlattices in the Chlorine Dioxide-Iodine-Malonic Acid Reaction-Diffusion System
}

\author{
Igal Berenstein, Lingfa Yang, Milos Dolnik, Anatol M. Zhabotinsky, and Irving R. Epstein* \\ Department of Chemistry and Center for Complex Systems, MS 015, Brandeis University, \\ Waltham, Massachusetts 02454-9110
}

Received: February 2, 2005; In Final Form: April 26, 2005

\begin{abstract}
We study the mechanism of development of superlattice Turing structures from photochemically generated hexagonal patterns of spots with wavelengths several times larger than the characteristic wavelength of the Turing patterns that spontaneously develop in the nonilluminated system. Comparison of the experiment with numerical simulations shows that interaction of the photochemical periodic forcing with the Turing instability results in generation of multiple resonant triplets of wave vectors, which are harmonics of the external forcing. Some of these harmonics are situated within the Turing instability band and are therefore able to maintain their amplitude as the system evolves and after illumination ceases, while photochemically generated harmonics outside the Turing band tend to decay.
\end{abstract}

\section{Introduction}

The study of pattern formation in nonequilibrium reactiondiffusion systems began with the theoretical analysis of Turing structures. Turing ${ }^{1}$ suggested that such structures could play a role in morphogenesis, and his point of view has gradually become prominent in theoretical biology. 2,3 The first experimental observation of Turing patterns, however, occurred nearly 40 years after Turing's work, in a chemical reaction-diffusion system. ${ }^{4}$ Later, Kondo and collaborators ${ }^{5-7}$ showed that skin patterns in various small fish also develop according to the Turing mechanism.

Superlattices are symmetric structures composed of several simple component patterns, such as hexagonal or square lattices, with appropriately related wavelengths. They were originally studied in solid-state physics and used in the development of semiconductor devices. ${ }^{8,9}$ In nonequilibrium systems, superlattice patterns are formed by Faraday waves. ${ }^{10-12}$ They have been found in other hydrodynamic, ${ }^{13}$ magnetohydrodynamic, ${ }^{14}$ and optical $^{15}$ systems. Superlattice patterns are seen on leopard and jaguar skins. Spontaneously emerging Turing structures are usually simple hexagonal lattices or stripe patterns. ${ }^{16}$ The only Turing superlattice that spontaneously arises from random initial conditions is the so-called "black eye". ${ }^{17,18}$

Recently, we discovered Turing superlattice structures that develop in the chlorine dioxide-iodine-malonic acid (CDIMA) reaction-diffusion system from simple hexagonal patterns created by illumination of the working layer through appropriate masks. ${ }^{19}$ A system that without illumination generates a labyrinthine stripe pattern is illuminated through a mask with a hexagonal or square pattern of transparent or opaque spots, resulting in the illumination pattern being imprinted on the system. After the illumination is turned off, this pattern evolves into a Turing superlattice if $R$, the ratio of the wavelength of the illumination pattern to the average wavelength of the natural labyrinthine pattern, is close to an integer. The superlattice preserves the wavelength of the illumination pattern.

Here we monitor and analyze the course of evolution from the photochemically produced simple lattices to the final superlattices. Comparison of our experiments with numerical

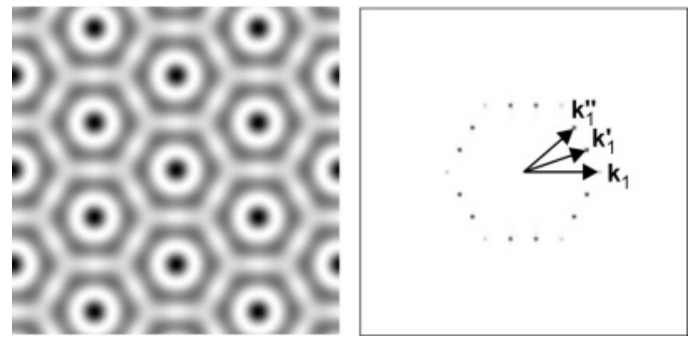

Figure 1. Hexagonal superlattice-3 (left) is a combination of three simple hexagonal lattices rotated by $20^{\circ}$, as seen in the Fourier transform at the right. It displays period-3 oscillations of the gray level along the principal translation axes.

simulations reveals the mechanism of superlattice formation. We show that interaction of the photochemical periodic forcing with the Turing instability results in generation of multiple resonant triplets of wave vectors, which are harmonics of the external forcing. Some of these harmonics lie within the Turing instability band and are able to maintain their amplitude after illumination ceases. They preserve high amplitude during evolution and form the framework of the superlattice. Some harmonics and subharmonics of these Turing modes have significant amplitude and modulate the shape of the elementary cells of the superlattice, while the amplitudes of most of the photochemically generated harmonics outside the Turing band decay virtually to zero.

\section{Experimental Section}

Details of the experimental setup have been described elsewhere. ${ }^{20,21}$ In brief, a continuously fed, unstirred, one-sided reactor with a $0.3 \mathrm{~mm}$ thick agarose gel layer as the working medium is fed with the reagents of the CDIMA reaction with initial concentrations $\left[\mathrm{I}_{2}\right]_{0}=0.37 \mathrm{mM},[\mathrm{MA}]_{0}=1.8 \mathrm{mM}$, $\left[\mathrm{ClO}_{2}\right]_{0}=0.14 \mathrm{mM}$, and $[\mathrm{PVA}]_{0}=10 \mathrm{~g} / \mathrm{L}$, where $\mathrm{MA}$ is malonic acid and PVA is poly(vinyl alcohol). The residence time of reagents in the feeding chamber is $230 \mathrm{~s}$. The temperature is $4.0 \pm 0.2{ }^{\circ} \mathrm{C}$. Under these conditions, a labyrinthine pattern with an average wavelength of $0.45 \mathrm{~mm}$ develops spontaneously. ${ }^{21}$ 


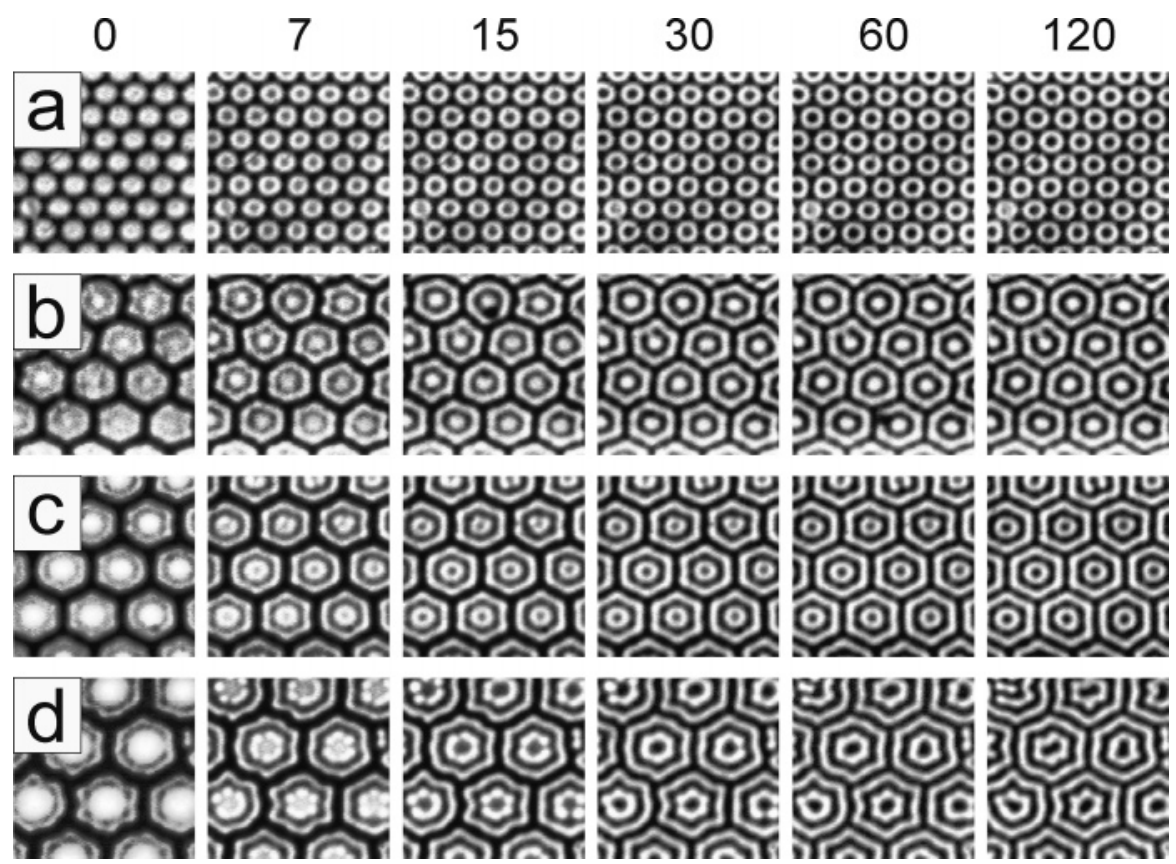

Figure 2. Development of hexagonal superlattices induced with transparent spot hexagonal masks with $R=$ (a) 2.0, (b) 3.0, (c) 4.0, and (d) 5.0. The first column displays patterns immediately after the end of the forcing illumination. The numbers above the columns show the time in minutes after cessation of illumination. Frame size is $5 \times 5 \mathrm{~mm}^{2}$.

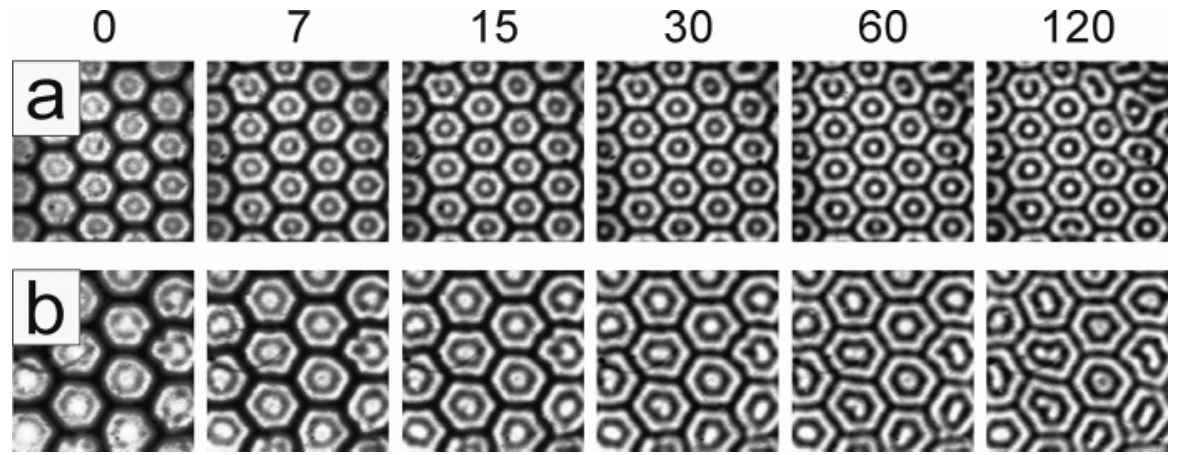

Figure 3. Development of hexagonal superlattices induced with transparent spot hexagonal masks with $R=$ (a) 2.5 and ( b) 3.2 . Frame size is 5 $\times 5 \mathrm{~mm}^{2}$.

After the labyrinthine pattern becomes stationary, the system is brought to a spatially uniform steady state with homogeneous illumination of intensity $70 \mathrm{~mW} / \mathrm{cm}^{2}$. Then the intensity is changed to $23.6 \mathrm{~mW} / \mathrm{cm}^{2}$, a mask is placed between the source light and the reactor, and the image of the mask is focused on the surface of the gel. We use masks with hexagonal patterns of spots with the transmittance of the masks determined by the sums of sinusoidal functions. We vary the ratio $(R)$ of the spatial period of the illumination pattern to the average period of the original labyrinthine pattern in the range $0.8-6.0$. The forcing illumination is turned off when the photochemically induced pattern becomes stationary. Snapshots are taken under a light intensity of $0.6 \mathrm{~mW} / \mathrm{cm}^{2}$ with a CCD camera.

\section{Model}

We employ a two-variable model that includes the effect of illumination: ${ }^{22-24}$

$$
\begin{gathered}
\frac{\partial u}{\partial t}=a-u-4 \frac{u v}{1+u^{2}}-w(\overrightarrow{\mathrm{r}})+\nabla^{2} u \\
\frac{\partial v}{\partial t}=\sigma\left[b\left(u-\frac{u v}{1+u^{2}}+w(\overrightarrow{\mathrm{r}})\right)+\mathrm{d} \nabla^{2} v\right]
\end{gathered}
$$

Here $u$ and $v$ are the dimensionless concentrations of $\left[\mathrm{I}^{-}\right]$and $\left[\mathrm{ClO}_{2}{ }^{-}\right]$, respectively; $a, b, d$, and $\sigma$ are dimensionless parameters. In our simulations $a=12, b=0.2, d=1$, and $\sigma=50$, which results in the formation of striped Turing patterns analogous to those obtained in the experiments. With $\left[\mathrm{ClO}_{2}\right]=$ $0.14 \mathrm{mM}, 1$ time unit (tu) corresponds to approximately $400 \mathrm{~s}$, and 1 space unit (su) to $0.07 \mathrm{~mm}$.

The term $w(\overrightarrow{\mathrm{r}})$ represents the photochemical reaction that consumes $\mathrm{I}^{-}$and produces $\mathrm{ClO}_{2}{ }^{-}$at a rate proportional to the intensity of illumination. As in the experiment, the illumination patterns are hexagonal lattices with the intensity of illumination determined by the sum of three sinusoidal functions chosen so that the minimum intensity is zero. The maximum intensity is designated $W$.

We carry out two-dimensional simulations of a square medium with zero-flux boundary conditions. In most of the simulations, the grid spacing is $0.5 \mathrm{su}$. Where necessary, this size is decreased 2- to 8-fold to ensure the desired precision. We employ the explicit Euler method with a time step of 6.25 $\times 10^{-4}$ tu or less. The system size is given in each figure legend.

\section{Results and Discussion}

Simple hexagonal lattices are formed by resonant triplets of vectors: $\mathbf{k}_{1}+\mathbf{k}_{2}+\mathbf{k}_{3}=0$, with equal wavenumbers. ${ }^{16}$ 


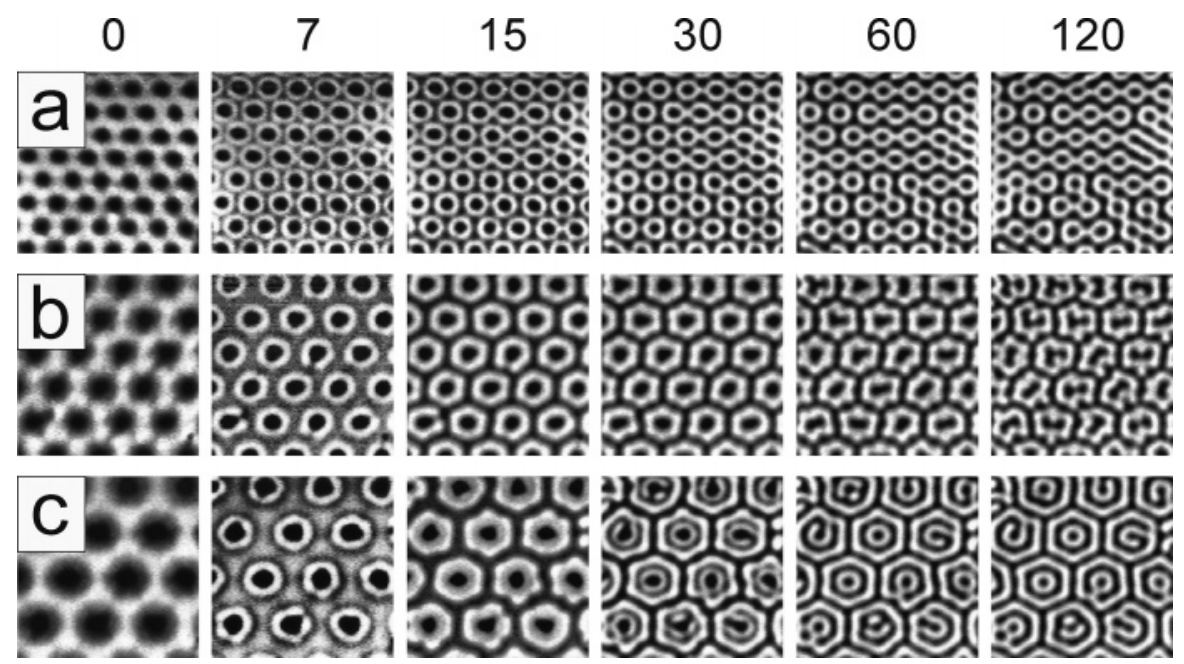

Figure 4. Development of hexagonal superlattices induced with opaque spot hexagonal masks with $R=$ (a) 2.0, (b) 3.0, and (c) 4.0. Frame size is $5 \times 5 \mathrm{~mm}^{2}$.

a)
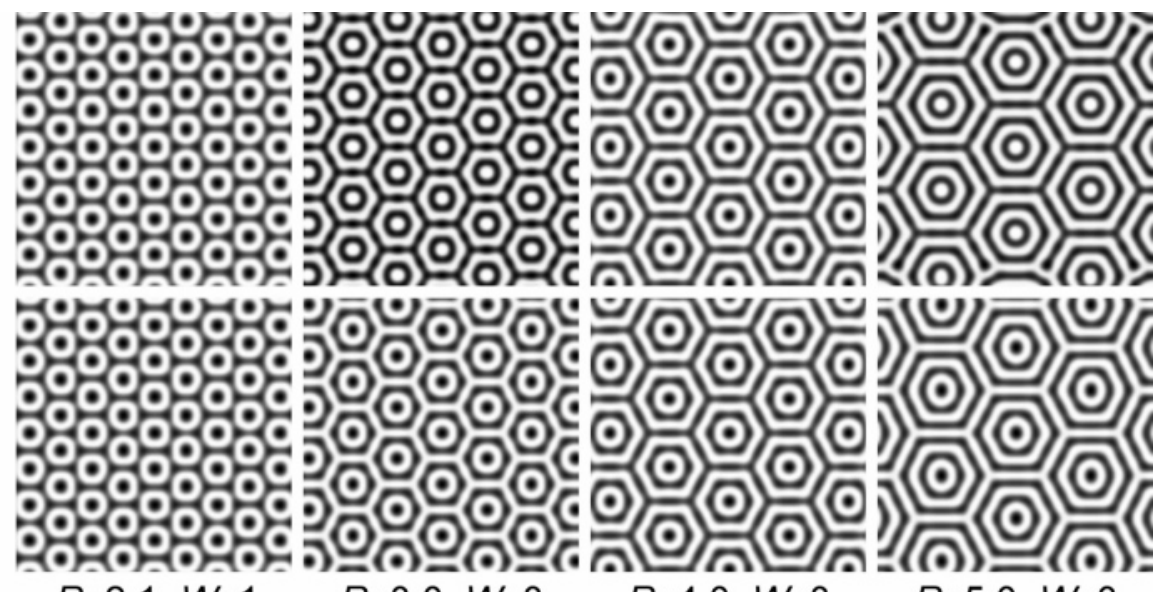

$$
R=2.1, W=1 \quad R=3.0, W=3 \quad R=4.0, W=3 \quad R=5.0, W=3
$$

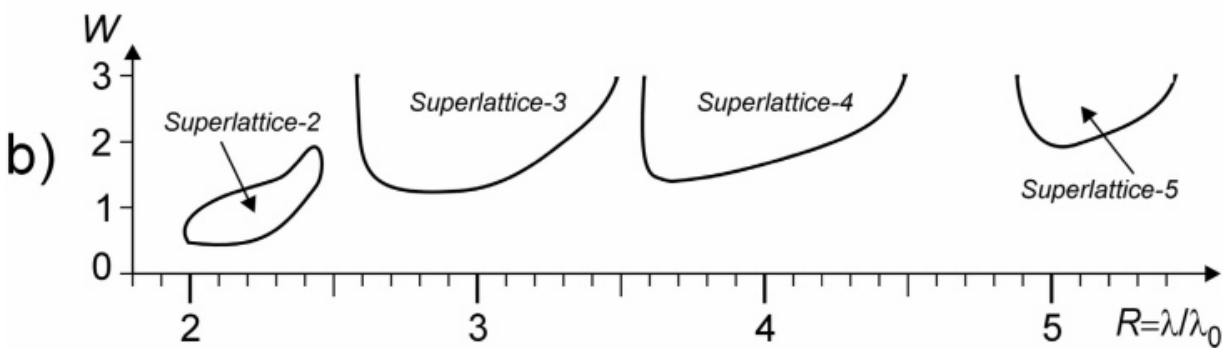

Figure 5. Stable hexagonal superlattices obtained in simulations with model (1). (a) Superlattices (after cessation of illumination) induced with patterns of illuminated spots on a dark background are in the top row; those induced with patterns of dark spots on an illuminated background are in the bottom row. Frame size is $128 \times 128 \mathrm{su}^{2}$. (b) Domains of existence of stable superlattices in the $(W-R)$ plane.

Superlattices consist of several such triplets, which are shifted in phase. Figure 1 shows a superlattice constructed from three simple lattices rotated by $20^{\circ}$, as revealed by the Fourier spectrum. This pattern contains oscillations of optical density along the principal translation axes, in which one spatial period contains three maxima. In what follows, a superlattice induced by illumination patterns with $R$ near an integer $N$ usually displays concentration oscillations along the principal translation axes in which one spatial period contains $N$ maxima. We designate such a pattern superlattice- $N$.

Figure 2 demonstrates the development of hexagonal superlattices induced with transparent spot masks. White corresponds to high chlorite and low iodide concentrations, black to the opposite situation. Illumination with $R=2.0$ and 3.0 results in superlattices- 2 and -3 , respectively, while $R=4.0$ and 5.0 both yield superlattice-4. Elementary cells in the pattern induced with $R=5.0$ display significant irregularities.

Figure 3 shows the development of hexagonal superlattices induced with transparent spot masks with $R=2.5$ and 3.2. Both patterns are superlattice-3, but they are less regular than the pattern induced with $R=3.0$ and start to deteriorate earlier than the latter.

Figure 4 demonstrates the evolution of hexagonal superlattices induced with opaque spot masks. Illumination with $R=2.0$ and 3.0 yields superlattice-2, while $R=4.0$ results in superlattice-4. These patterns have more defects than the patterns induced with transparent spot masks and soon start to lose the symmetry of the elementary cells.

Figure 5a shows stable hexagonal superlattices obtained in simulations with model (1). At a fixed duration of illumination 

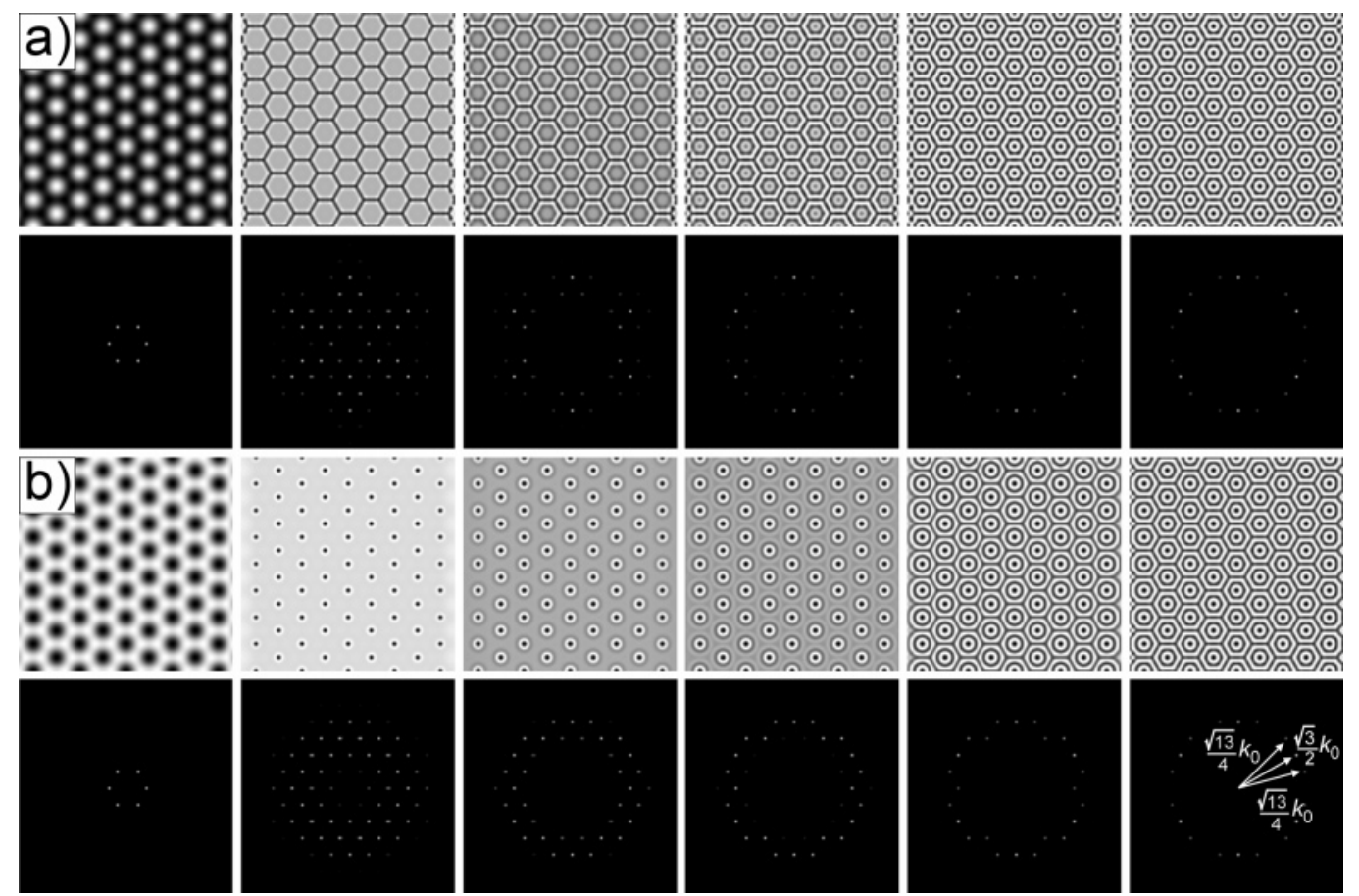

Figure 6. Simulations of development of hexagonal superlattices after forcing with hexagonal illumination patterns of (a) transparent and (b) dark spots with $R=4.0$, model (1). The first column shows the illumination patterns (first and third rows), which were applied for 30 tu, and their Fourier spectra (second and fourth rows). The following columns show the induced patterns and their Fourier spectra at 1, 3, 5, 10, and 20 tu after the end of illumination. The frame size is $256 \times 256 \mathrm{su}^{2}$.
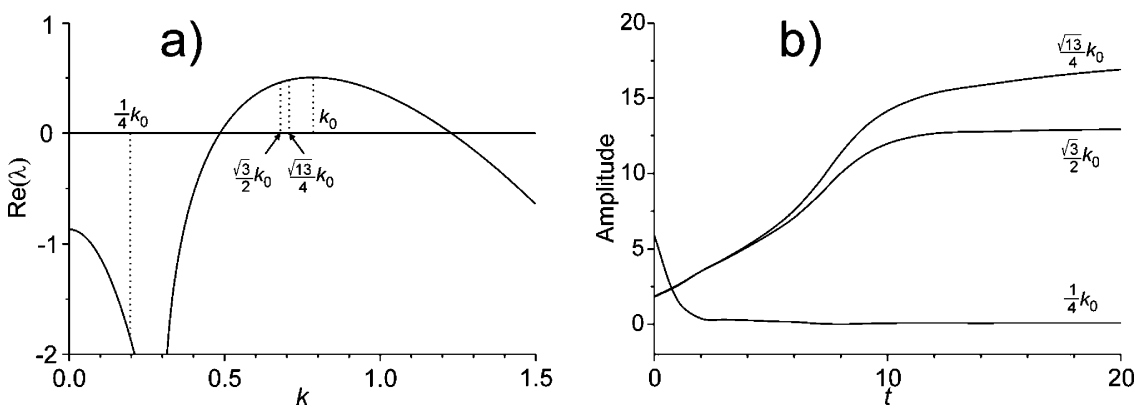

Figure 7. (a) Dispersion relation calculated with eq 1 for the stable superlattice- 4 in Figure 6 . The wavenumber of the most positive Turing mode, $k_{0}$, is 0.8 . The wavenumber of the illumination pattern $(R=4.0)$ is $k_{0} / 4$. Of the three principal hexagonal modes one has $k=(\sqrt{13} / 4) k_{0}$ and two others $k=(\sqrt{3} / 2) k_{0}$. (b) Growth kinetics of the three principal modes with wavenumbers inside the Turing band, and decay of the illumination mode situated outside.

stable superlattices-2 can be induced in a closed domain in the $R, W$-plane (Figure $5 \mathrm{~b}$ ). The domains of induction of stable superlattices-3, -4 , and -5 seen in the figure are tongues whose $R$-ranges grow as $W$ increases.

Figure 6 shows simulations of the development of hexagonal superlattices after forcing with hexagonal illumination patterns with $R=4.0$. The sinusoidal illumination patterns in the first column have a single spatial frequency. The second column shows the induced patterns and their Fourier spectra at $1 \mathrm{tu}$, immediately after the end of illumination. The Fourier spectra reveal that interaction of the photochemical periodic forcing with the Turing instability results in generation of multiple resonant triplets of wave vectors, which are harmonics of the external forcing. The two illumination patterns generate different sets of transient spatial harmonics, which ultimately yield the same pattern. As the system evolves, only harmonics situated within the Turing instability band grow. The amplitudes of other harmonics decrease drastically, so they are barely visible in the spectra in the last column of Figure 6. Linear stability analysis of eq 1 gives the dispersion relation shown in Figure 7a, which confirms that the wavenumbers of the principal vectors of the superlattice (cf. bottom right panel of Figure 6) lie within the Turing band. Figure $7 \mathrm{~b}$ shows the kinetics of growth of the relevant modes and the rapid decay of a mode outside the Turing band.

The simulations display good qualitative agreement with the experiments. They produce superlattices with the same patterns of elementary cells when $R$ is close to an integer. The superlattices preserve the periods of the illumination patterns and the positions of their elementary cells. Illumination with transparent spots yields elementary cells with black centers in even-numbered superlattices and with white centers in odd ones. Illumination with opaque spots always results in black centers. Thus inversion of the illumination pattern leads to inversion of the resulting superlattice at odd $N$, but makes no difference at even $N$.

The major discrepancy between our model simulations and the experiments lies in the stability of the patterns. The superlattice patterns in the model are stable. To confirm the stability, established superlattices were subjected to global perturbation by small amplitude spatial noise for times of the order of the time for development of the superlattice. The noise 


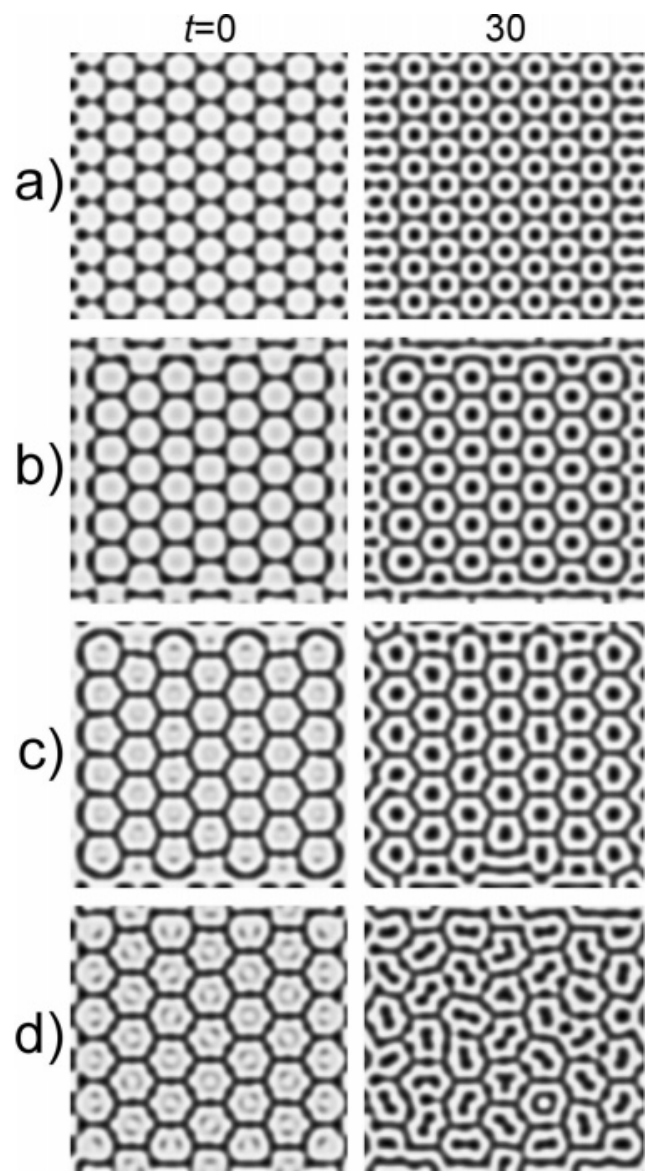

Figure 8. $R$-dependence of the elementary cell shape in simulated superlattices-2. $R=2.0$ (a), 2.2 (b), 2.4 (c), and 2.6 (d). The superlattices were induced with hexagonal patterns of transparent spots with $W=$ 1.0 and 30 tu illumination. Frame size is $128 \times 128 \mathrm{su}^{2}$.

generated visible defects in the superlattice patterns, but below a threshold noise amplitude the perturbed patterns relaxed back to the original superlattices after removal of the noise. Thus, the superlattices are stable in the Lyapunov sense. If the noise amplitude was sufficiently large, defects in the superlattices did not vanish after removal of the noise but continued to grow. In some cases the distorted superlattices evolved into labyrinthine patterns. Our simulations show that superlattice patterns are stable in closed domains of the model parameters. These domains are typically separated by regions where only labyrinthine patterns are stable.

In the experiments, superlattices start to deteriorate sooner or later depending on their type. Inspection of Figures 2c, 3, and 4 reveals the interplay of the effect of wavelength, which is known to result in the zigzag instability of parallel stripes, ${ }^{25}$ and that of curvature. These effects determine both the shape of the elementary cells and their stability with respect to symmetry-breaking perturbations. Superlattices-2 display a richer behavior. The experiments (Figures 2 and 4) and simulations (Figure 8) show that with increasing $R$ the external boundary of the white rings changes from almost circular to hexagonal. When $R$ lies to the left of the superlattice- 2 stability domain, connections develop between white rings, leading in some cases to the appearance of straight lines of connected circles (Figures $4 \mathrm{c}$ and $9 \mathrm{a}$ ). Later, the white rings start to open, eventually evolving to stripes. When $R$ is to the right of the superlattice- 2 stability domain, the central black spots start to elongate and gradually turn into irregular striped segments. Meanwhile, the external boundaries of the white hexagons become concave, then lose hexagonal symmetry and eventually become irregular (Figures $4 \mathrm{~b}$ and $9 \mathrm{~b}$ ). For superlattices of higher order only the latter type of evolution has been observed. Examples are shown in Figures 2d, 3b, and 9c.

One possible cause of deterioration of the theoretically stable superlattices in our experiments is spatial noise resulting from imperfections in the gel layer. Three-dimensional effects may also play a role, since the thickness of the gel layer is about 0.67 of the fundamental wavelength.

Our experiments and simulations demonstrate that at the same parameters either superlattices or labyrinthine patterns can emerge depending on the initial conditions. This is indicative of a subcritical bifurcation leading to the formation of the superlattice. On the other hand, "black eye" patterns can emerge spontaneously from random initial conditions. This suggests that superlattices of the third or higher order might also arise spontaneously via supercritical bifurcations in rather narrow parameter domains.

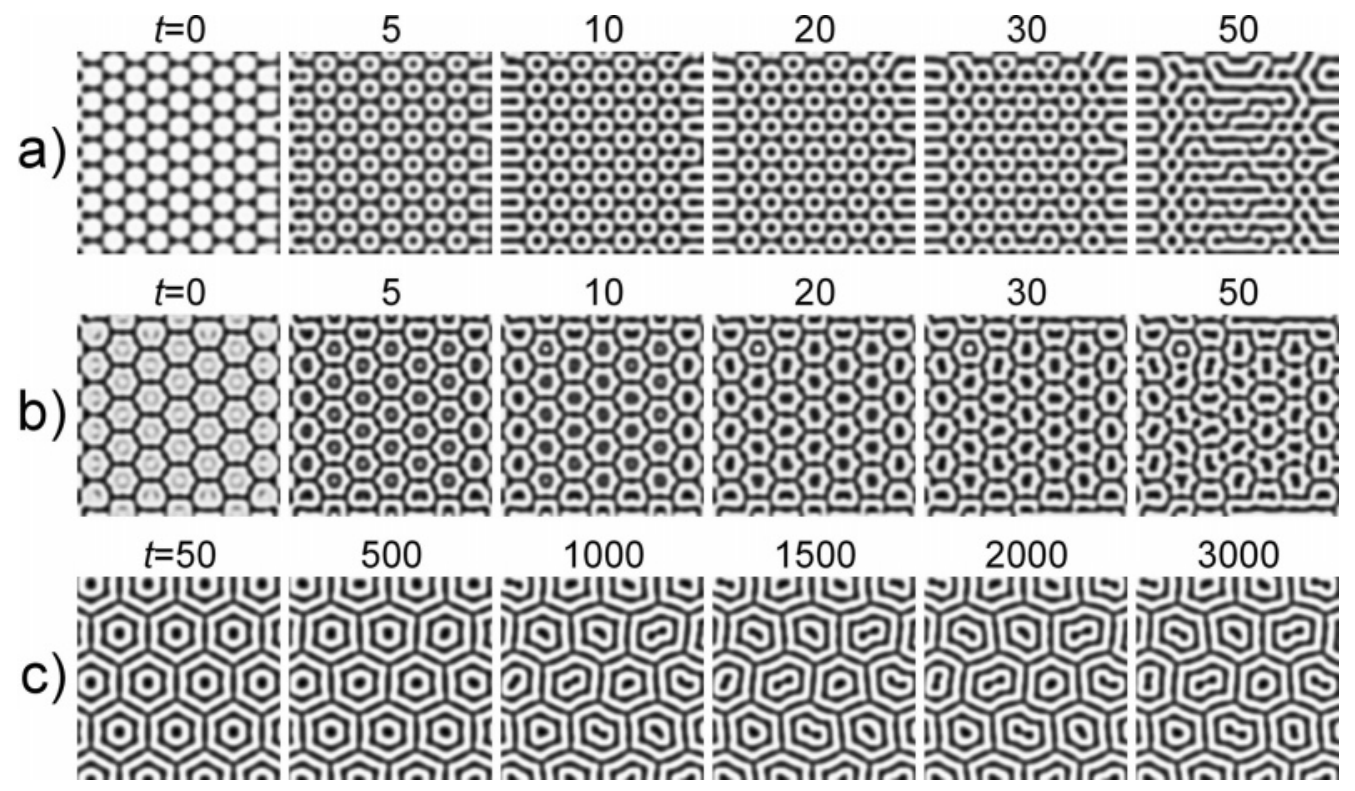

Figure 9. Examples of evolution of superlattices induced with hexagonal patterns of transparent spots with 30 tu illumination outside the stability domains in the $(R, W)$-plane: (a) $R=2.0, W=1.0$; (b) $R=2.6, W=1.0$; and (c) $R=4.6, W=3.0$. Frame size is $128 \times 128 \mathrm{su}^{2}$. 
We induced superlattices by creating the necessary prepatterns photochemically. It is also possible to generate initial conditions of the type used in this work in a more "natural" way. For instance, in two-layer asymmetric systems, ${ }^{26}$ one layer could generate a simple hexagonal lattice, which could then induce a superlattice pattern in a second layer.

Acknowledgment. This work was supported by grant CHE0306262 from the National Science Foundation.

\section{References and Notes}

(1) Turing, A. M. Philos. Trans. R. Soc. London B 1952, 237, 37.

(2) Meinhardt, H. Models of Biological Pattern Formation; Academic: London, UK, 1982. 1989.

(3) Murray, J. D. Mathematical Biology; Springer: Berlin, Germany,

(4) Castets, V.; Dulos, E.; Boissonade, J.; De Kepper, P. Phys. Rev. Lett. 1990, 64, 2953.

(5) Kondo, S.; Asai, R. Nature 1995, 376, 765.

(6) Asai, R.; Taguchi, E.; Kume, Y.; Saito, M.; Kondo, S. Mech. Devel. 1999, $89,87$.

(7) Kondo, S. Genes Cells 2002, 7, 535 .

(8) Smith, D. L.; Mailhiot, C. Rev. Mod. Phys. 1990, 62, 173.

(9) Braun, P. V.; Osenar, P.; Stupp, S. I. Nature 1996, 380, 325.
(10) Kudrolli, A.; Pier, B.; Gollub, J. P. Physica D 1998, 123, 99.

(11) Arbell, H.; Fineberg, J. Phys. Rev. Lett. 1998, 81, 4384.

(12) Arbell, H.; Fineberg, J. Phys. Rev. E 2002, 65, 036224.

(13) Rogers, J. L.; Schatz, M. F.; Brausch, O.; Pesch, W. Phys. Rev. Lett. 2000, 85, 4281

(14) Pi, H. J.; Park, S.; Lee, J.; Lee, K. J. Phys. Rev. Lett. 2000, 84, 5316.

(15) Vorontsov, M. A.; Samson B. A. Phys. Rev. A 1998, 57, 3040.

(16) Kapral, R.; Showalter, K., Eds. Chemical Waves and Patterns; Kluwer: Dordrecht, The Netherlands, 1995.

(17) Gunaratne, G. H.; Ouyang, Q.; Swinney, H. L. Phys. Rev. E 1994, $50,2802$.

(18) Zhou, C.; Guo, H.; Ouyang, Q. Phys. Rev. E 2002, 65, 036118.

(19) Berenstein, I.; Yang, L.; Dolnik, M.; Zhabotinsky, A. M.; Epstein,

I. R. Phys. Rev. Lett. 2003, 91, 058302.

(20) Dolnik, M.; Berenstein, I.; Zhabotinsky, A. M.; Epstein, I. R. Phys. Rev. Lett. 2001, 87, 238301.

(21) Berenstein, I.; Dolnik, M.; Zhabotinsky, A. M.; Epstein, I. R. J. Phys. Chem. A 2003, 107, 4428.

(22) Lengyel, I.; Epstein, I. R. Science 1991, 251, 650.

(23) Rudovics, B.; Barillot, E.; Davies, P. W.; Dulos, E.; Boissonade, J.; De Kepper, P. J. Phys. Chem. A 1999, 103, 1790.

(24) Horvath, A. K.; Dolnik, M.; Muñuzuri, A. P.; Zhabotinsky, A. M.; Epstein, I. R. Phys. Rev. Lett. 1999, 83, 2950.

(25) Passot, T.; Newell, A. C. Physica D 1994, 74, 301.

(26) Berenstein, I.; Dolnik, M.; Yang, L.; Zhabotinsky, A. M.; Epstein, I. R. Phys. Rev. E 2004, 70, 046219. 